\title{
Testing Device Drivers against Hardware Failures in Real Environments
}

\author{
Satoru Takekoshi \\ University of Tsukuba \\ 1-1-1 Tennodai \\ Tsukuba \\ Ibaraki 305-8577 Japan \\ satorut \\ @osss.cs.tsukuba.ac.jp
}

\author{
Takahiro Shinagawa \\ The University of Tokyo \\ 2-11-16 Yayoi \\ Bunkyo-ku \\ Tokyo 113-8656 Japan \\ shina@ecc.u-tokyo.ac.jp
}

\author{
Kazuhiko Kato \\ University of Tsukuba \\ 1-1-1 Tennodai \\ Tsukuba \\ Ibaraki 305-8577 Japan \\ kato@cs.tsukuba.ac.jp
}

\begin{abstract}
Hardware failures are a major cause of computer system crashes. To improve system reliability, making device drivers sufficiently robust to tolerate hardware failures is important. Unfortunately, testing device drivers against hardware failures is not easy because hardware failures must be artificially produced in a controlled manner. Prior work has required source code, OS-dependent implementation efforts, or special virtual environments for testing in which it is difficult to find problems that only occur in real environments. This paper presents FaultVisor, a bare-metal hypervisor specialized for testing device drivers. FaultVisor injects pseudo hardware failures by modifying access to real hardware using the hypervisor layer, allowing closed-source and OS-independent driver testing in real environments, and easy insertion of test systems into existing systems. In our evaluation, FaultVisor discovered 41 fault patterns that disclose problems in device drivers, 30 of which led to critical system failures.
\end{abstract}

\section{CCS Concepts}

-Computer systems organization $\rightarrow$ Reliability;

\section{Keywords}

Device Driver; Operating System; Virtualization; Reliability

\section{INTRODUCTION}

The reliability of computer systems has become more important as the dependency on computer systems has increased. Computer system failures can result in massive financial or life loss, even with only tens of minutes of downtime. Hardware failures are a major cause of system crashes [4]. Unfor-

Permission to make digital or hard copies of all or part of this work for personal or classroom use is granted without fee provided that copies are not made or distributed for profit or commercial advantage and that copies bear this notice and the full citation on the first page. Copyrights for components of this work owned by others than ACM must be honored. Abstracting with credit is permitted. To copy otherwise, or republish, to post on servers or to redistribute to lists, requires prior specific permission and/or a fee. Request permissions from permissions@acm.org.

SAC 2016, April 04-08, 2016, Pisa, Italy

(C2016 ACM 978-1-4503-3739-7/16/04 .. \$15.00

DOI: http://dx.doi.org/10.1145/2851613.2851740

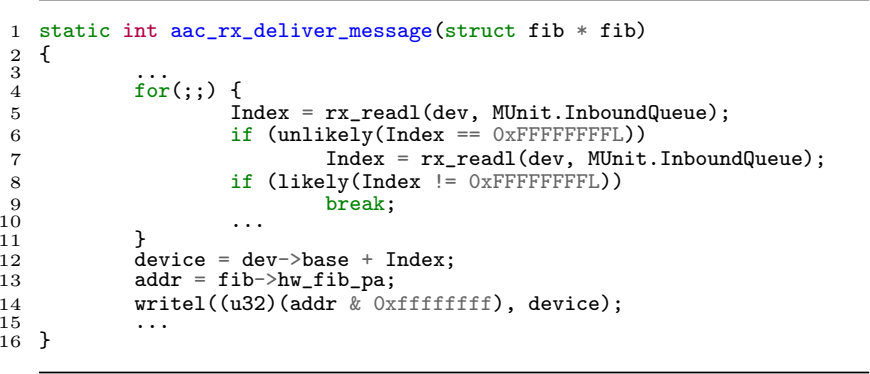

Figure 1: Example of unreliable code. An index variable received from the hardware in line 5 or 7 is used for pointer arithmetic in line 12 only if it is not equal to OxFFFFFFFF (aacraid in Linux 4.0; rx.c).

tunately, with the wide spread of low-cost commodity hardware products, hardware failures have become inevitable. Therefore, to improve reliability, computer system software should tolerate hardware failures as much as possible.

Device drivers are the software components that directly interact with hardware devices. The developers of device drivers often assume that hardware devices always function as expected. However, hardware devices sometimes behave unexpectedly when transient or permanent failures, such as bit-flip failures and stuck-at failures [10], occur. If device drivers do not expect such hardware failures, the drivers cannot work correctly, which leads to system crashes. For example, the code fragment shown in Figure 1, which is in a RAID controller driver from Linux 4.0, uses a value received from a device control register (line 5 or 7 ) as an index value to access another memory-mapped register (line 12 and 14). This code does not check the range of the index value received from the hardware, thereby causing potential illegal memory access beyond boundaries when the hardware returns an unexpected value due to failure. A robust device driver should check such values to avoid system crashes.

Device drivers are known to be the weakest components of operating systems. A study into operating system errors 12 reported that Linux drivers have an error rate up to seven times higher than the rest of the kernel. In Windows Vista, millions of crashes were caused by device drivers 13 . More- 
over, many device drivers are vulnerable to hardware failures. A study by Microsoft [11 has shown that $9 \%$ of unplanned reboots of Windows servers were due to driver or hardware failures, and the majority of such failures were transient. That study also showed that fault tolerant systems could decrease storage and network adapter failure rates from $8 \%$ to $3 \%$, and they claimed that drivers could mask the effects of device failure by design. Such evidence implies that (1) poorly implemented drivers are widely used in products and (2) drivers can survive some hardware failures if properly implemented.

A previous study used source code analysis to find inappropriate handling of hardware failures 4]. Unfortunately, this approach cannot test closed-source drivers and has the possibility of false detection. Several studies have used symbolic execution to explore the entire code for testing [5, 6 , 7. However, this requires special virtualized environments and can miss side-effect behavior that only occurs in real environments.

This paper presents FaultVisor, a bare-metal hypervisor for testing device drivers against hardware failures via fault injection. FaultVisor does not virtualize devices and allows pass-through access to real hardware, while only slightly modifying access to the hardware to simulate hardware failures. In cooperation with controller software running in user mode, target devices and fault patterns can be configured easily during runtime. In addition, testing can be performed repeatedly and automatically, and the test cases are reproducible. FaultVisor is transparent from the guest OS and kernels, and drivers do not require modification. It is also easily inserted into existing systems in order to test device drivers in real environments.

We have implemented FaultVisor based on BitVisor 3, which is a versatile platform for mediating device access for various purposes $1,[2]$. In our evaluation, FaultVisor and a manual analysis based on the test results identified 41 fault patterns that disclose problems. Thirty of the identified patterns caused critical system failures, such as crashes or hangs.

\section{RELATED WORK}

Driver reliability has been a significant concern for computer systems. Therefore, numerous studies with various approaches have been conducted to address this problem, some of which have targeted hardware failures.

\subsection{Source Code Analysis}

Source code analysis is a common approach for finding bugs. Carburizer [4] analyzes the source code of Linux drivers to find the locations where drivers incorrectly trust hardware behavior. Carburizer can automatically insert recovery mechanisms into existing drivers. Unfortunately, Carburizer requires source code and cannot be applied to closed-source drivers. Furthermore, Carburizer may give false detections when drivers have input validation schemes that it cannot detect. FaultVisor can be applied to closed-source drivers and does not give false detections come from the static structure of the program.

\subsection{Symbolic Execution}

Symbolic execution is a software testing technique that explores every possible software execution path to observe possible behaviors. DDT [5], S ${ }^{2} \mathrm{E}[6]$, and SymDrive 7] employ symbolic execution for driver testing. Although they can achieve good code coverage, they also have the following limitations. First, they run drivers in specialized virtual environments. Computer system environments consist of many software and hardware components and configurations that affect each other. Symbolic execution can miss side-effect behavior that only occurs in real environments because the testing environments for symbolic execution differ from real environments. For example, some previous work has required a limitation on the number of CPU cores for a guest OS. FaultVisor does not require such changes and covers such behavior because it tests drivers in nearly equivalent environments as the final products. Second, symbolic execution requires OS-specific implementation, including kernel patches. FaultVisor is minimally OS-specific and requires no kernel patches. Therefore, it is easily applicable to various OSs and versions.

\subsection{Stress Testing}

Microsoft provides Driver Verifier [14, which is a stresstesting tool for Windows device drivers. Driver Verifier can perform various tests against drivers, such as the Low Resources Simulation test or Force Pending I/O Requests test; however, it primarily tests against interfaces in the Windows system and does not perform fault injection to test device drivers against hardware failures.

\subsection{Runtime Fault Tolerance}

Runtime fault tolerant systems for device drivers, such as SafeDrive 8] and Nooks 9], execute device drivers in an isolated environment. Isolation can confine the effects of hardware failures in a limited space, thereby avoiding complete system crashes. However, they require OS-specific implementation and runtime overhead to protect device drivers.

\section{DESIGN}

The goal of FaultVisor is to test device drivers against hardware failures in real environments without expensive OSspecific work. To achieve this goal, we have designed FaultVisor to require no special driver or modifications to existing OS kernels and drivers. In most cases, configurations do not require changes. Figure 2 shows an overview of the FaultVisor testing platform. The platform is divided into two parts, i.e., a hypervisor that runs under the OS and a controller that runs within the userland of the OS.

\subsection{Hypervisor}

To inject faults, the hypervisor slightly modifies access to hardware from device drivers based on a given configuration. This configuration includes target hardware devices, the I/O addresses of devices, and fault modes. The hypervisor intercepts I/O read access to the target devices, modifies their values based on the fault mode, and passes the values to the device drivers. To improve OS transparency, the hypervisor does not modify access to hardware devices that are not specified in the configuration. To improve testing 


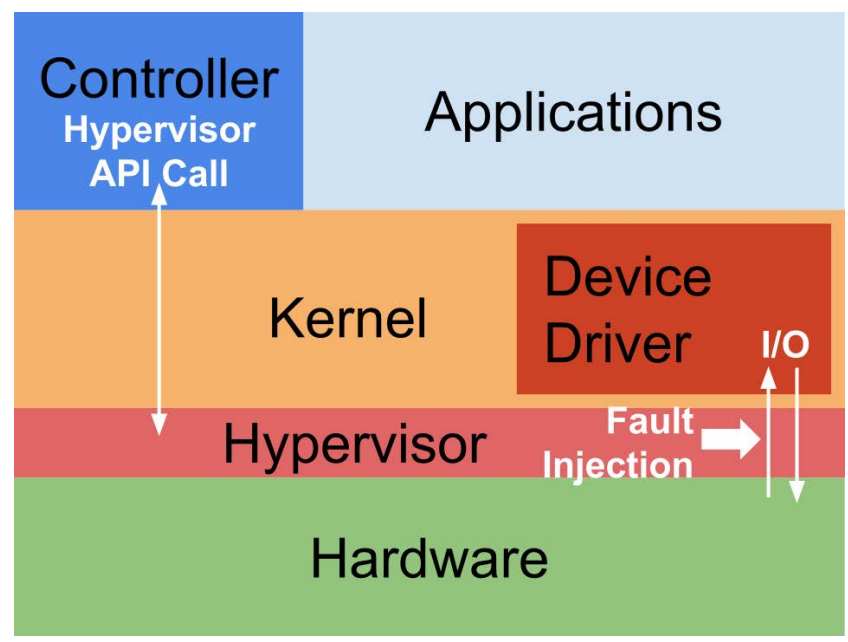

Figure 2: Overview of FaultVisor. A hypervisor injects faults to simulate hardware failures by slightly modifying access to hardware from device drivers. A controller running in user mode communicates with the hypervisor to determine target devices and fault patterns.

flexibility, the hypervisor provides a set of APIs that can be called from user mode to configure and control testing.

Device driver misbehavior can cause system hangs that cannot be recovered by OS kernels. To recover from such situations and continue testing, the hypervisor monitors the status of the guest OS and invokes a forced reboot when it detects a system hang.

\subsection{Controller}

The controller is responsible for controlling the overall testing. Its functions include (1) notifying configurations to the hypervisor, (2) starting and stopping testing, (3) invoking device driver functions to explore execution paths, and (4) logging events that occur in the OS. The controller first determines the configuration, and then notifies the configuration to the hypervisor via API calls. This improves flexibility because test patterns can be programmable with user-mode applications. The controller also starts and stops testing by calling APIs.

Invocation of driver functions must be performed within the OS because drivers typically work in response to system calls from user-mode applications. The controller executes some applications and performs operations as programmed to explore the execution paths in the drivers. The controller observes events that occur in the OS and collects logs. To continue testing on sudden reboots caused by OS crashes or hangs, we configure the OS to execute the controller automatically on startup. The controller detects and logs abnormal termination of a test. To investigate the situation further, the controller can repeat the test by reproducing questionable events.

\section{IMPLEMENTATION}

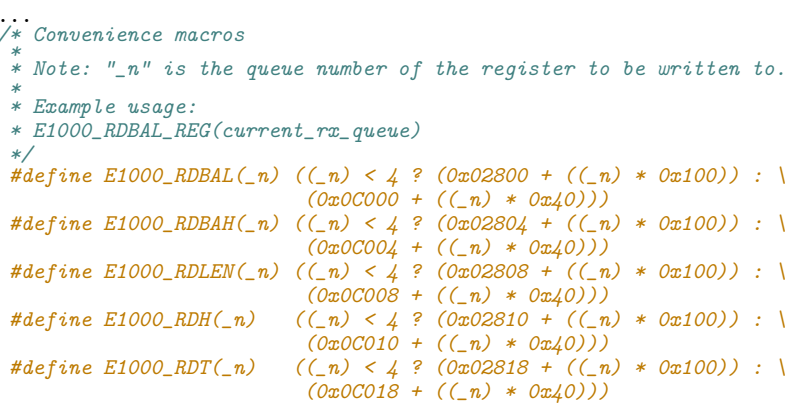

Figure 3: Complex I/O region definition (e1000e 3.1.0.2; regs.h)

\subsection{Hypervisor}

The hypervisor is implemented based on BitVisor [3], which is a lightweight bare-metal hypervisor. BitVisor is opensource and has been used in several studies [1,2]. BitVisor does not require a host OS and starts execution as part of the boot process in the bootloader. It chainloads the bootloader of a guest OS and exposes the same interface with the one of bare-metal. This characteristic allows us to significantly reduce the side effects of virtualization.

\subsubsection{Target $\mathrm{I} / \mathrm{O}$ access}

Our target hardware devices are PCI/PCIe devices accessed via port-mapped I/O (PIO) or memory-mapped I/O (MMIO). We can easily intercept PIO access through processor virtualization functions such as Intel VT-x and AMD-V. MMIO can be intercepted with nested paging such as Intel EPT or AMD NPT. Note that we currently do not modify direct memory access (DMA). Device drivers typically treat DMA data as opaque and do not interpret their content. Therefore, DMA data does not affect the behavior of device drivers in many cases. However, we believe that we can apply our approach to DMA, if required.

The hypervisor receives the device information to be monitored from the controller. This information includes bus numbers and the device numbers of PCI/PCIe devices. The hypervisor looks for the PCI configuration space of the specified devices and collects device information such as PIO and MMIO address regions.

Based on the $\mathrm{I} / \mathrm{O}$ regions, the hypervisor intercepts and modifies I/O access. However, modifying every I/O access to the I/O regions usually results in OS crashes in the early stage of driver initialization, thereby leaving most execution paths untested. Thus, we must repeat the test for subsets of the I/O regions. Unfortunately, device drivers usually use only a limited range of $\mathrm{I} / \mathrm{O}$ regions, and testing all $\mathrm{I} / \mathrm{O}$ regions is generally a waste of time. For open-source drivers, we can learn the used I/O regions from the source code. However, this requires human effort, especially when the source code is complex. For the example shown in Figure 3 the $\mathrm{I} / \mathrm{O}$ regions are defined with complex macros and are difficult to interpret. Moreover, if a driver is closed-source, it is impossible to know the I/O regions. 




Figure 4: Minimal controller code for flipping all bits in $\mathrm{I} / \mathrm{O}$ requests for a register of the Intel PRO/1000 network adapter

To determine the used I/O regions with minimal effort, we have implemented a two-pass method. In this method, we use a monitor mode to monitor I/O access from device drivers and determine the I/O region used by the given driver. After the first pass, we read log information from the hypervisor to determine the I/O regions. Then, we switch the hypervisor to a one of fault-injection modes so that it can modify parts of the used I/O regions.

\subsubsection{Fault-injection modes}

We have implemented two fault modes in the hypervisor, i.e., xormask and fixed. Xormask performs exclusive-or (XOR) operations against the original values in I/O access. The mask value for XOR operations is specified by the controller. For example, if the controller specifies 0x0001 as an XOR mask value, the least significant bit of the value in the $\mathrm{I} / \mathrm{O}$ access will be flipped. Xormask mode produces controlled pseudo bit-flip errors. Fixed is a mode in which some bits in the value of the I/O access are fixed to a specified value. For example, if the controller specifies 0x0001 as a fixed value, the least significant bit is fixed to 1 . The parameters of each mode can be specified for each I/O address.

\subsubsection{Hang-up detection}

The hypervisor detects OS hangs by monitoring ping from the controller, which is not the network one but the one performed using the VMCALL instruction 15] trapped by the hypervisor. VMCALL is a general instruction that calls a hypervisor from the guest OS, thereby causing a forced transition from guest mode to hypervisor mode. VMCALL can be executed in non-privileged user-mode processes. We use VMCALL for communication between the hypervisor and the controller. The controller periodically sends pings to the hypervisor using VMCALL to notify that the guest OS is alive. Simultaneously, the hypervisor periodically gets control with the preemption timer of Intel VT-x even if the guest OS is completely stuck. When the guest OS is stuck for a certain period, the hypervisor forcibly reboots the machine. This mechanism is portable among many OSs.

\subsection{Controller}

Reducing human effort is essential for driver testing. We have implemented a small testing framework for writing a controller in Python. We can write a controller with a small
Table 1: List of tested drivers

\begin{tabular}{|c|c|c|}
\hline Driver & $\mathrm{OS}$ & Source code \\
\hline \multirow{2}{*}{$\begin{array}{c}\text { Adaptec RAID } 5805 \\
\text { (RAID controller) }\end{array}$} & Linux & open \\
\hline & Windows & closed \\
\hline \multirow{2}{*}{$\begin{array}{l}\text { Marvell 88SE9123 } \\
\text { (SATA controller) }\end{array}$} & Linux & open \\
\hline & Windows & closed \\
\hline \multirow{2}{*}{$\begin{array}{c}\text { NVIDIA GT } 610 \\
\text { (GPU) }\end{array}$} & Linux & closed \\
\hline & Windows & closed \\
\hline
\end{tabular}

amount of code using this framework. Figure 4 shows minimal controller code that flips all bits in the I/O access for the MMIO register 0x0000 of an Intel PRO/1000 device. We have implemented a mechanism to execute VMCALL in Python scripts with the Python extension for C.

As a result of this test, the controller outputs the performed fault injection patterns (i.e., a pair of fault mode and its parameter such as an XOR mask value or a fixed value) and events observed from the controller during the testing of each pattern. Developers and debuggers can analyze and reproduce the discovered problems with the log. In addition, they can inject other faults patterns in their debugging, if required.

\section{EVALUATION}

\subsection{Driver Testing}

We tested six device drivers for three types of devices with FaultVisor. The tested drivers with the corresponding device names are shown in Table 1 All evaluations were performed with Lubuntu 14.04.2 LTS (Linux kernel 3.16) and Windows Server 2012 running on a machine with an Intel Core i7 4770K processor and 16 GB RAM.

The evaluations for each driver included the following steps:

1. Choose one register from the I/O register set.

2. Set a random value as the fault pattern and initiate register fault-injection.

3. Load the driver into the OS kernel.

4. Invoke driver function by application.

5. Attempt to unload the driver.

6. Determine if the driver is stuck or the device is in an incorrect state.

(a) If so, reboot the machine and continue.

7. Return to 1 and select the next register.

Register sets were acquired using the FaultVisor monitor mode. We used practical applications to invoke driver functions. For example, we executed a GPGPU application from the CUDA Toolkit Code Samples, vectorAddDrv, to invoke the NVIDIA GPU driver. To check the state of the driver and device, we examined the log output or attempted to load and unload the driver without any fault-injection and 
Table 2: Numbers of registers

\begin{tabular}{|c|c|c|}
\hline Driver & Used & Tested \\
\hline \multicolumn{3}{|c|}{ Linux } \\
\hline Adaptec RAID 5805 & 2058 & 10 \\
\hline Marvell 88SE9123 & 59 & 59 \\
\hline NVIDIA GT 610 & 81476 & 533 \\
\hline \multicolumn{3}{|c|}{$\overline{\text { Windows }}$} \\
\hline Adaptec RAID 5805 & 16395 & 11 \\
\hline Marvell 88SE9123 & 9 & 9 \\
\hline NVIDIA GT 610 & 65305 & 729 \\
\hline
\end{tabular}

observed if it succeeded. Tests were performed with both xormask and fixed mode. All processes were performed automatically using the programmed controller.

Since the NVIDIA GPU and Adaptec RAID drivers used too many MMIO registers, we excluded some of the registers from the test target to perform the tests efficiently. We found that these drivers read many consecutive registers as if they had executed memcpy from MMIO regions and such registers account for large portion of all registers. Therefore, we decided to exclude registers that were consecutive over 256 bytes. The number of MMIO registers tested is shown in Table 2. Note that no driver used PIO registers.

\subsection{Discovered Problems}

We examined abnormal events occurred during the tests, such as sudden reboots or kernel errors, and performed manual analysis by reproducing such abnormal events under FaultVisor. We also considered cases that did not cause immediate crashes or hangs as problems if they met the following criteria. Such problems were usually due to drivers that failed to detect hardware failures and misbehaved.

Linux the kernel or driver outputs an error or warning message with stack dumps into the kernel message buffer (e.g., failed on sanity check by the WARN_ON macro or a soft-lockup).

Windows the driver stopped abnormally but was recovered by the OS (only for the display driver).

Table 3 summarizes the number of fault patterns found with FaultVisor. Here, "Faults" is the number of fault patterns that disclosed problems. "Critical" is the number of fault patterns that led to system crashes or hangs. "Tests" is the number of tests performed. "Time" is the total test time in (hour):(minute) format. One test count means that we performed a fault-injection test for each target register once in both xormask and fixed modes. When we found fault patterns that disclose problems on the same register in both xormask and fixed modes, we counted it as one fault pattern.

As shown in Figure 1, aacraid, the Linux driver for Adaptec RAID 5805, uses a value read from the device in pointer arithmetic without proper range checks, thereby leading to system crashes on hardware failures. Another fault pattern for aacraid is passing an incorrect argument from the driver
Table 3: Number of fault patterns that disclosed problems

\begin{tabular}{|c|c|c|c|c|}
\hline \multirow{2}{*}{ Driver } & \multicolumn{1}{|c|}{$\begin{array}{c}\text { Faults } \\
\text { Linux }\end{array}$} & Time \\
\cline { 3 - 3 } & & Critical & \\
\hline \hline \multicolumn{5}{|c|}{ Linux } \\
\hline Adaptec RAID 5805 & 3 & 2 & 3 & $01: 12$ \\
\hline Marvell 88SE9123 & 0 & 0 & 3 & $01: 56$ \\
\hline NVIDIA GT 610 & 10 & 3 & 1 & $04: 33$ \\
\hline \hline \multicolumn{5}{|c}{ Windows } \\
\hline Adaptec RAID 5805 & 2 & 2 & 3 & $03: 00$ \\
\hline Marvell 88SE9123 & 1 & 1 & 3 & $00: 33$ \\
\hline NVIDIA GT 610 & 25 & 22 & 1 & $11: 20$ \\
\hline
\end{tabular}

to a kernel function. These are the two critical fault patterns listed in Table 3

The Marvell 88SE9123 (ahci) Linux driver appears robust; however, we found that the Windows counterpart (storahci. sys) was vulnerable to hardware failure. Windows causes a Stop error (Blue Screen of Death) with code DPC_WATCHDOG_ VIOLATION when values read from an MMIO register are bit-flipped by XOR with a specific value. We cannot confirm the root cause of this hang because storahci.sys is closed-source. However, we suppose that this fault is tolerable by software if properly implemented because ahci does not cause hangs with the same fault pattern and operation. storahci.sys is the standard AHCI SATA controller bundled with Windows Server 2012 and Windows 8.

We found several fault patterns that make NVIDIA GPU drivers misbehave. For the Linux driver (nvidia), we observed various errors such as "divide error", "NULL Pointer Dereference" or "Bad RIP value". Some faults allowed system survival; however, the system hung when rebooted. For the Windows driver, we saw common Stop error codes such as DRIVER_IRQL_NOT_LESS_OR_EQUAL or SYSTEM_THREAD_ EXCEPTION_NOT_HANDLED. We classified faults that caused hangs or crashes without any human operation after the fault-injection test as critical. We stopped the evaluation for NVIDIA GPU drivers after one test because driver tests are time consuming and we had identified a sufficient number of fault patterns.

As mentioned previously, FaultVisor found several fault patterns that disclosed problems in device drivers. In this evaluation, we did not depend on any device-specific knowledge and did not alter any kernel code. This indicates that FaultVisor might be able to find more fault patterns if combined with other approaches.

\subsection{False Patterns}

We experienced several strange hangs in 12 fault patterns in the NVIDIA GPU driver tests. In this case, the hypervisor could not reboot the machine. We think this was caused by genuine transient hardware failures for the following reasons: (1) physical power and reset buttons did not work, (2) the hypervisor became almost unresponsive, and (3) MachineCheck Exception (i.e., an exception reporting a hardware error) raised in one hang case. 




Figure 5: Ping latency of Intel I218V.

This type of hang was classified as propagative hardware failure; a driver failure is propagated into the device (e.g., the driver read a fault-injected value and wrote it to the device). Potentially, device drivers can tolerate such failures by detecting failures and preventing fault propagation to the device. However, they are out of scope of our current test and not included in Table 3

We automated recovery from such hangs by using an Arduino 17 connected to the motherboard. The Arduino was programmed to monitor the HDD access indicator and perform a forced electrical reboot when the indicator stopped blinking for a specified period.

In addition to hangs, during NVIDIA GPU driver tests, we found 10 fault patterns that did not reproduce bad events or result in hangs in userland applications. These fault patterns may cause driver failures under rare conditions. We also excluded them from the results.

The false fault patterns mentioned above were all found in the NVIDIA GPU driver tests. These cases and the large number of their registers (Table 2) suggest that the drivers and hardware are very complicated. Although FaultVisor can cope with such drivers, as shown in Table 3 , some false cases are inevitable with our current approach.

\subsection{The Effects of Virtualization}

FaultVisor exposes bare-metal hardware to the guest OS. This design reduces the effects of the virtualization significantly. However, there will be some inevitable effects of virtualization. To evaluate the effects, we carried out zero XOR tests. In the test, FaultVisor intercept all I/O between the driver and the device and flips bits of I/O with xormask mode. XOR mask value in the test is fixed to zero so that no pseudo hardware failures will be injected, while FaultVisor perform the interception. All tests were performed in the environment of subsection 5.1

First, we performed zero XOR tests for a sanity check. We performed the tests for every driver tested in subsection 5.1 for at least one hour with the workload. As a result, we did not observe any errors or abnormal behavior of OSs or drivers.

Second, we performed zero XOR tests for overhead measurements of FaultVisor. We carried out performance evalu-

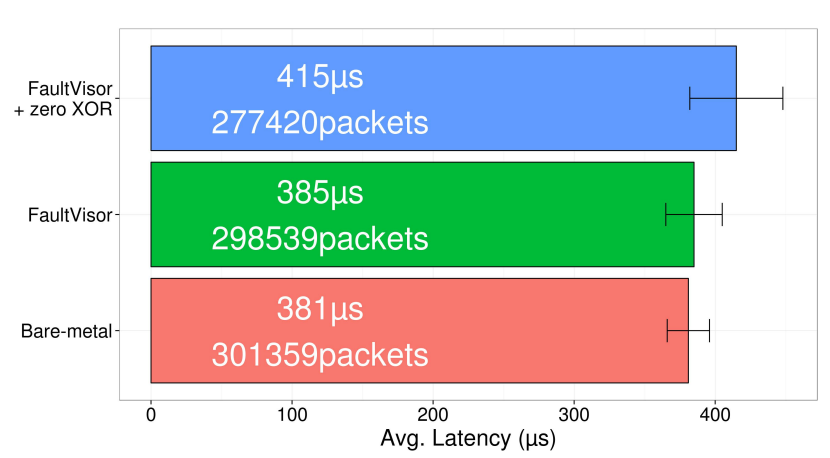

Figure 6: Ping latency of Realtek RTL8111GR.

ation of two network interface cards (Intel I218V and Realtek RTL8111GR) with FaultVisor and compared the result with those of bare-metal and FaultVisor without zero XOR. In the evaluation, we measured the latency with the $\operatorname{ping}(8)$ utility and the throughput with the netperf(1) by connecting two machines with a network cable directly. All measurements were run for 120 seconds. Figure 5 and Figure 6 shows the results of the latency measurements. It suggests that I/O interception by FaultVisor incurs overhead on the latency. This extent of increased network latency can occur due to environmental differences. We did not find any significant difference in the throughput measurements between FaultVisor and bare-metal.

The results of these tests imply that FaultVisor itself does not make a significant difference for the guest OSs and drivers compared to bare-metal. Furthermore, latency among MMIO pages would not differ significantly because all MMIO pages for the target device were intercepted and processed through the same execution path in FaultVisor. Therefore, FaultVisor does not affect driver mechanisms such as MMIO timing calibration.

\section{LIMITATIONS AND FUTURE WORK}

The current implementation of FaultVisor has several limitations. First, FaultVisor only runs on Intel processors. Second, FaultVisor does not test failures that occur on erroneous interrupts. Third, FaultVisor does not test against faulty DMA. Further development efforts will remove these limitations and make FaultVisor more useful.

When a device has non-volatile memory (NVM), the driver can corrupt its content in the tests by FaultVisor. For example, it can happen when driver writes fault-injected value back to NVM-related registers. Corrupted NVM can lead to the problems such as permanent failure of the device. We avoid this by excluding access to NVM-related registers from the tests target.

As the goal in this work is to test drivers in unmodified environments, we did not patch any kernel code in the evaluation. In addition, we left most configurations unchanged. This reduces the amount of possible event logs collected during the tests. In the current implementation, we collect logs such as those from the Linux kernel ring buffer. The user 
can patch the kernel code or change configurations to obtain more verbose logs during tests and it will support the analysis of the problems. Further analysis of the discovered problems with such modifications is a future work.

Fault patterns, such as bit flipping patterns, are generated randomly in our current implementation. Though this approach discovered several problems in reasonable time, there should be more efficient method for faults generation which stands on the detailed fault studies.

\section{CONCLUSION}

Device drivers can survive some hardware failures if the failures are detected properly. Unfortunately, device drivers that do not expect hardware failures are widely used, which can lead to system crashes and hangs. Although several studies have tested drivers against hardware failures, none supports testing closed-source device drivers in real baremetal environments in which device drivers run as a part of final products. This paper has presented FaultVisor, a bare-metal hypervisor specialized for fault-injection testing of device drivers. FaultVisor produces controlled pseudo hardware failures. FaultVisor is easily inserted into existing systems to perform fault-injection tests in real environments. We tested six drivers using FaultVisor with Linux and Windows and found 41 fault patterns that disclose problems, including 30 patterns that led to system crashes or hangs.

\section{REFERENCES}

[1] Y. Omote et al. "Improving Agility and Elasticity in Bare-metal Clouds." In Proc. ASPLOS 2015, 145-159, Mar. 2015.

[2] Y. Matsuhashi et al. Transparent VPN Failure Recovery with Virtualization. Future Generation Computer Systems, Elsevier, Vol. 28, No. 1, pp. 78-84, Jan 2012.

[3] T. Shinagawa et al. "BitVisor: A Thin Hypervisor for Enforcing I/O Device Security." In Proc. VEE 2009, pp. 121-130, Mar 2009.

[4] A. Kadav et al. "Tolerating Hardware Device Failures in Software." In Proc. SOSP 2009, pp. 59-72, Oct 2009.

[5] V. Kuznetsov et al. "Testing Closed-Source Binary Device Drivers with DDT." In Proc. USENIX Annual Technical Conference, 2010.

[6] V. Chipounov et al. "S2E: A Platform for In Vivo Multi-Path Analysis of Software Systems." In Proc. ASPLOS 2011, pp. 265-278, 2011.

[7] M. Renzelmann et al. "SymDrive: Testing Drivers without Devices." In Proc. OSDI 2012, pp. 279-292, 2012.

[8] F. Zhou et al. "SafeDrive: Safe and Recoverable Extensions Using Language-Based Techniques." In Proc. OSDI 2006, pp. 45-60, 2006.

[9] M. Swift et al. "Improving the Reliability of Commodity Operating Systems." ACM TOCS, 23(1), Feb. 2005

[10] M. Li et al. "Understanding the Propagation of Hard Errors to Software and Implications for Resilient
System Design." In Proc. ASPLOS 2008, pp. 265-276, Mar. 2008.

[11] S. Arthur. "Fault resilient drivers for Longhorn server", WinHec 2004 Presentation DW04012, 2004.

[12] A. Chou et al. "An Empirical Study of Operating Systems Errors." In Proc. SOSP 2001, pp. 73-88, Oct. 2001.

[13] Microsoft. Microsoft internal memo, disclosed as public evidence in court case 2:07-cv-00475-MJP, Kelley v. Microsoft Corporation Filing 131. https://docs.justia.com/cases/federal/district-courts/ washington/wawdce/2:2007cv00475/142597/131 2008.

[14] Microsoft Driver Verifier. https://msdn.microsoft. com/en-us/library/windows/hardware/ff545448.aspx

[15] Intel@64 and IA-32 Architectures Software Developer Manuals http: //www.intel.com/content/www/us/en/processors/ architectures-software-developer-manuals.html

[16] Nouveau: Accelerated Open Source driver for nVidia cards http://nouveau.freedesktop.org/

[17] Arduino http://www.arduino.cc/ 